
\title{
25 Research Soure \\ Providers' Definitions of Quality and Barriers to Providing Care: a Qualitative Study in Rural Mpumalanga Province, South Africa
}

Rebecca L West ( $\sim$ rlwest@bu.edu )

Boston University https://orcid.org/0000-0002-2958-0884

Sheri A Lippman

University of California San Francisco

Rhian Twine

University of the Witwatersrand

Meriam Maritze

University of the Witwatersrand

Kathleen Kahn

University of the Witwatersrand

Hannah H Leslie

Harvard University

Research

Keywords: Nurses, Quality, Indicators, Primary Health Care, Qualitative Research, South Africa

Posted Date: August 6th, 2020

DOl: https://doi.org/10.21203/rs.3.rs-52850/v1

License: (1) This work is licensed under a Creative Commons Attribution 4.0 International License.

Read Full License 


\section{Abstract}

Background: Facing a quadruple burden of disease (infectious disease, non-communicable disease, maternal and child mortality, high levels of violence and injury), South Africa requires high-quality primary health care to retain patients and optimize outcomes. National health policy is focused on strengthening primary care. While prior research has identified implementation challenges within the primary health care system, there is less understanding of how providers define quality, their perceptions of barriers to providing quality care, and how they overcome these barriers. This study assesses provider views on quality at primary care clinics in a rural region of Mpumalanga Province.

Methods: We conducted in-depth interviews with providers in Bushbuckridge sub-district in early 2019 on the value of quality metrics for providers and patients, what indicators they would use to assess clinic performance, and barriers and facilitators of delivering care. Interviews were conducted in Shangaan, audio-recorded, translated, and transcribed into English. A deductive approach was used to develop a provisional coding schema based on study questions, which was refined using an inductive approach in response to patterns and themes emerging from the data.

Results: 23 providers were interviewed (83\% female, $65 \%$ professional nurses). Definitions of quality were focused on clinic structure and resources. Few providers identified patient outcomes as indicators of quality. Providers linked deficiencies in infrastructure and support to deficits in care delivery, such as long wait times due to limited staffing, privacy breaches due to insufficient space, and a chronic lack of medication and equipment. Providers identified mitigating strategies including informal coordination across clinics to address medication shortages in individual facilities. Interwoven throughout the providers' discussion was the poor communication between the district, PHC supervisors, and implementers at the facility level.

Conclusion: Providers connected deficits in quality of care to inadequate infrastructure and insufficient support from district and provincial authorities; mitigating strategies across clinics could only partially address these deficits. The existence of a national quality measurement program was not broadly reflected in providers' views on quality care. These findings underscore the need for effective district and national approaches to support individual facilities, accompanied by feedback methods designed with input from frontline service providers.

\section{Introductions}

South Africa currently faces a quadruple burden of disease driven by coexisting infectious diseases (HIV/AIDS, tuberculosis), non-communicable diseases (vascular illness, diabetes, cancer), avertable maternal and child mortality, and high levels of violence and injuries (1-4) that collectively place a heavy burden on the primary health care (PHC) system.(5) The South African government is organized into three levels: national, provincial, and district.(5) PHC is provided through the district health system, and health sector governance is centered within the provincial health departments, while funding and policy 
guidelines are made at the national level.(6) Decentralization of health care has increased access to healthcare facilities, but has also intensified problems of disparity in poor, rural areas (7), leading to lowquality health care delivery. This is particularly the case in areas that were pre-apartheid Bantustans which have historically suffered from underfunding and lack of resources. $(8,9)$ While the South African government aims to provide universal health coverage, it must also ensure high quality of care across the public health system to reap its benefits. $(10,11)$

High quality health care is important for retaining patients and optimizing outcomes for those in need of continuous clinical services. High-quality health systems consistently provide care that improves or maintains health, are valued and trusted by all people, and respond to changing population needs.(12) The foundations of high-quality health systems include the population and their health needs and expectations, governance of the health sector and partnerships across sectors, platforms for care delivery, workforce numbers and skills, and tools and resources; these foundations inform quality processes of care which lead to quality impacts.(12)

The South African health system is rooted in the concept of PHC (6), which is provided primarily by professional and enrolled nurses in clinics and community health centers (CHCs).(5) Services offered in PHC facilities include maternal and child care, immunization, family planning, syndromic treatment of sexually transmitted infections, HIV testing and counseling, and care for chronic diseases. CHCs operate 16-24 hours a day, providing additional maternity services and accident and emergency services. Clinics typically offer services 8-9 hours a day.(5) Both CHCs and clinics offer services seven days a week.

The national government has introduced numerous policy reforms and initiatives $(13,14)$ including the Ideal Clinic Realization and Maintenance (ICRM) program (15) to improve quality of PHC services.(11) ICRM works to support facility-level quality improvement through provision of manuals and training as well as a district support team. However, poor communication between national government, international funders and policy developers, and poor oversight of actual PHC service delivery, continues to create deficits in the performance of district health systems. $(8,11)$ In 2016 , a modeling study suggested that of the estimated 97,000 preventable deaths in South Africa, 51,000 (53\%) were attributable to poor quality of care, through incorrect management or inability to retain the patient in care, among those utilizing the health system.(16)

Research assessing overall health system quality and patient experience has identified numerous implementation challenges and deficiencies within the PHC system, including unequal distribution of resources, management and leadership $(2,9,17)$; service delivery issues such as long wait times $(2,8,18)$; and poor hygiene and infection control.(2,19) A number of studies have documented providers' insights into challenges in providing quality care across sub-Saharan Africa. $(5,17,20-24)$ Missing from the current literature is an understanding of what providers perceive as quality care, particularly situated within a framework of high quality health systems defined by the ICRM framework and the Lancet Global Health Commission on High-Quality Health Systems. $(11,12)$ We conducted a qualitative research study within a resource-constrained, rural South African setting to identify what providers define as quality care 
and the barriers they face in providing quality care. This research can identify gaps in the existing foundations of care needed to provide quality services, and define priorities to advocate for resources and improve quality care at the provider level. $(25,26)$

\section{Methods}

\section{Study Setting}

The Agincourt Health and Socio-Demographic Surveillance System (HDSS) research area is operated by the Medical Research Council (MRC)/Wits University Rural Public Health and Health Transitions Research Unit (Agincourt) in the rural Bushbuckridge sub-district of Ehlanzeni District in Mpumalanga Province. The Agincourt HDSS is located about 500 kilometers northeast of Johannesburg, near the border of Mozambique, and is home to roughly 115,000 individuals living in 31 contiguous villages.(27) Within the Agincourt HDSS, approximately 1 in 5 adults is living with HIV (28), over half of adults 40 years and older have elevated blood pressure, and $10 \%$ have diabetes.(29) As of 2019, there are nine health facilities in the study area (three $\mathrm{CHCs}$ and six government satellite clinics). Three referral hospitals are situated 25 and 45 kilometers from the study setting.(27)

The MRC/Wits-Agincourt Unit maintains a longitudinal household census of area residents' sociodemographic status, and hosts a range of research studies, including clinical trials and cohort studies. (27) This research on quality of care was nested within a larger study being conducted in the site on community mobilization for HIV treatment as prevention, described elsewhere.(30) We conducted a crosssectional qualitative study among health care workers active in public health facilities within the Agincourt HDSS study area.

\section{Study Population}

Health care providers were purposively sampled from the nine PHC facilities in the Agincourt HDSS. Inclusion criteria for providers included being over 18 years of age and currently employed as a professional nurse, enrolled nurse, or lay HIV counsellor at a health facility within the Agincourt HDSS. The three CHCs were staffed by 14 to 25 nurses and 4 to 7 lay counselors, while the six clinics had between 6 and 14 nurse positions and 1 or 2 lay counselors. In order to reflect facility and staff size in our sample, at the three larger 24-hour CHCs, we aimed to interview three providers, while we aimed to interview two to three providers at the six smaller clinics.

\section{Data Collection and Analysis}

Data collection took place between February and May 2019. Interviews were held in person at the providers' clinic, in an area of their choice, and were approximately one hour in length. The interview guide was written in English, translated into the local language, Shangaan, and back translated in order to be reviewed by all members of the study team. The interview guide included open-ended questions eliciting provider perspectives on the value of health care quality metrics for providers and patients, what 
indicators they use to measure clinic performance, and barriers and facilitators of delivering quality care. Interviews were conducted by an individual experienced in conducting qualitative research in the Agincourt HDSS study area, who had completed high school and was fluent in Shangaan. Interviews were audio-recorded, translated, and transcribed into English. The study manager and qualitative interviewer reviewed all transcripts together in English for clarity prior to finalizing and coding them.

Transcripts were uploaded into NVivo qualitative analysis software (QSR International Pty Ltd. Version 12). A deductive approach was used to develop a provisional coding schema based on study questions. We used the high-quality health systems framework (12) to preliminarily identify barriers to care. Donabedian's domains of quality care (31) were also used to categorize indicators of care. A sub-set of transcripts was coded using this provisional schema in conjunction with an inductive approach that identified patterns and themes emerging from the transcripts.(32) The codebook was then reviewed and revised by members of the research team. All transcripts were coded using the finalized codebook by the study manager (RW). Illustrative statements of themes and sub-themes are provided and have been deidentified for inclusion in this report.

This research was approved by the Human Research Ethics Committee at the University of the Witwatersrand, the Mpumalanga Department of Health Research Committee and the Human Research Protection Program at Harvard University.

\section{Results}

We completed qualitative interviews with 23 participants from 9 facilities in the Agincourt HDSS, $82.6 \%$ of whom were female, which is representative of the healthcare workforce in this area. The majority of providers were professional nurses (65\%), with 5 enrolled nurses and 1 lay counselor. All providers approached to participate provided consent, although some did have to leave interviews early or reschedule based on clinical demands.

Providers discussed many barriers to providing quality care, which fell under the foundational categories of workforce and tools. These themes and related subthemes are discussed below.

\section{Barriers to providing quality care}

\section{Understaffing undermines provider capacity}

Providers discussed how understaffing diminished their ability to provide quality care through the creation of bottlenecks in service delivery, as well as the impacts of understaffing on their health and wellbeing. Almost all providers spoke of how they did not have enough staff working in their facility, particularly a lack of nurses but also filing clerks. Professional nurses were often required to take on duties such as checking vital signs that could be performed by enrolled nurses if they were available. Providers described how they had to hurry through patient visits and provide sub-optimal care, and some 
revealed they sometimes told patients to simply return the following day due to shortage of staff. As one provider said:

According to policy and guidelines it says, 'All chronic patients should have their urine taken and tested every visit.'But due to shortages, it becomes impossible... All chronic patients should be assessed fully from head to toe on a daily basis. How long would they wait if we practice that? (Clinic 4, professional nurse)

Providers discussed challenges with scheduling leave days and staffing throughout the week; weekends and Mondays were routinely understaffed despite high patient volume. Providers noted that there was no staffing buffer in the event of patient emergencies that occupied more experienced staff or for routine gaps such as lunch breaks, provider illness, or maternity leave. Providers were often forced to complete duties outside of their scope of work, such as collecting files and cleaning the clinics, as a result of these shortages:

On weekends there is no data capturer. The nurses have to do all the work... We don't have cleaners. We don't have grass cutters. As nurses we have to see that the yard is clean and we also have to clean the clinic. Again, we are the ones who have to retrieve files. This is taking our time. (Clinic 4, professional nurse)

Another scheduling issue was that of lay counsellors, whose hours are shorter than typical facility hours (weekday mornings only) as they are not part of the formal employment system of the Department of Health, and are meant to provide HIV testing services (HTS) on a part-time basis.(33) As a result, patients who come for HTS outside of the lay counsellors' hours have to be seen by a professional nurse.

Many providers discussed their health and wellbeing suffering as a result of understaffing, with specific mentions of depression, elevated stress, physical pain, exhaustion, and interpersonal or marital problems. One noted that these detriments to providers' wellbeing made them feel their own health was not a priority of the Department of Health. Another provider discussed how she had difficulty taking her own HIV medication due to lack of time to eat:

The shortage of staff is a serious problem at this clinic... we cannot take the lunch or breakfast breaks that we need as we are on treatment. If we carry on like this, we will collapse as this treatment requires us to eat now and again. (Clinic 9, professional nurse)

\section{Staff development approaches can be ineffective and counterproductive}

Providers discussed how the current approaches to training and supervision were ineffective and sometimes counterproductive. While providers identified a need for additional skills training, many raised issues with the in-service training model, in which senior providers were called to attend workshops and were responsible for relaying information back to staff. Providers reported concerns about the accuracy and comprehensiveness of information being conveyed back to them, if at all. The burden of trainings and under-staffing at healthcare facilities were intersecting issues. Providers discussed being chronically 
understaffed because nurses were at trainings or workshops, and one provider stated that they opted not to go to workshops as they knew it would leave facility staff overwhelmed. Providers also mentioned that being understaffed when they returned to the facility meant they did not have time to disseminate information learned in training. Providers cited a need for additional skills training, including care for patients with tuberculosis and HIV, as well as training on new medications:

You find that treatment is there but we don't know how to use it. You find that the treatment comes with a pamphlet and we have to read it. But it would be better if someone was there to demonstrate it to us. Seeing it is better than reading... We all need trainings when it comes to treatment. (Clinic 5, professional nurse)

The majority of providers also discussed issues with support and supervision at their facility, primarily at the district level. Providers said that when they did receive district supervision, it was overly critical and demotivating. Some felt that district supervisors only came when there was a serious issue at the clinic and were only there to "point fingers" or "shout and make noise." Providers expressed dissatisfaction with the way performance bonuses were given by the district. Some discussed simply not receiving the performance bonuses they were promised, or feeling that bonuses are distributed unfairly. Some providers spoke about how lay counsellors, who provide HTS, had recently gone on "go-slow" (working reduced hours and only serving a set number of patients) to demand their performance bonuses, which they felt had not been fairly distributed.

\section{Facility infrastructure and limited space impact ability to provide care}

Providers revealed how problems with facility infrastructure and limited space impacted their ability to provide quality care. Some providers interpreted poor facility infrastructure as an indication of the government's lack of concern for its constituents. Descriptions of inadequate space included insufficient meeting rooms, cramped service rooms, overcrowded reception areas with limited space for patients to wait comfortably, and lack of shelter for patients who had to wait outside. Structural issues in and around the clinic also impacted providers' ability to provide quality care; providers cited worries about their safety and patient safety due to issues such as lack of secure fencing, leaking roofs, and, in one clinic, a bat infestation in a collapsed roof. Periodic electricity outages were also noted in several clinics. Providers at one clinic described how the lack of filing cabinets jeopardized patient confidentiality, and providers avoided opening new files to save space.

Poor infrastructure also had a significant impact on confidentiality of patient care. Providers were concerned that patients could see or hear what was going on in consultation rooms because of the facility layout and size; in three facilities consultation rooms were separated only by a curtain. Providers mentioned having to take patients' vital signs in the waiting area, where they could not guarantee privacy. Another said there may often be two providers in one room seeing patients at the same time. Others were concerned that the facility layout made it difficult for patients to maintain confidentiality after testing for HIV: 
If you cry, that side, they will hear you. If you come out, there is no other door for you to use when coming out. The doors are looking at each other and people will see that you have a problem. The infrastructure is the problem. (Clinic 5, professional nurse)

Providers also cited issues with water and sanitation, including lack of clean water, broken toilets, and deficient cleaning materials, as impacting quality of care. Providers reporting issues with their water supply also claimed that the municipal water tanker responsible for delivering their water did not come regularly. Without water and proper cleaning materials, custodial staff (or providers, in clinics without custodial staff) could not clean the facility:

[The clinic] is dirty and smells bad. You cannot say it is clean by looking at it. You cannot wash the floor just with water. You need to get soap that can kill bacteria. It is easy for people to get infected with tuberculosis here as the place is not clean. (Clinic 5, professional nurse)

\section{Lack of equipment and medication reduces ability to provide quality care}

Providers discussed how insufficient equipment impacted their ability to provide competent care. Specific items mentioned include hemoglobin meters, beds with stirrups, wheelchairs, incubators, a child's scale, diapers, an autoclave to sterilize equipment, pregnancy tests, otoscopes, batteries, linen savers, HIV rapid test kits, and air conditioning units (important for optimal storage of medication).

The lack of equipment and/or faulty equipment caused delays in patient care, wasting patients' time and making visits longer as providers had to share equipment.

Providers discussed lacking medication, including cough medicine, diphtheria and tetanus vaccines, injectable contraceptives, blood pressure medication, and antiretroviral therapy (ART). Providers linked these issues to problems with deliveries of medication from the Mpumalanga Department of Health. They reported that orders placed with the medication depot were not fulfilled, fulfilled late, or fulfilled in different quantities than ordered. One provider linked this routine shortage of chronic medications (i.e. blood pressure medication, ART) contributing to patients' poor adherence:

Sometimes they come and you see that this person is really sick, but there is no treatment... Sometimes we are going to the nearest clinic to ask but also those clinics have limited treatment for their patients. We are worried about this issue. We keep on reporting and tell [the Department of Health] what we have done, but still they will tell you that the depot doesn't have treatment at the moment. (Clinic 2, enrolled nurse)

\section{Indicators of quality care}

Providers provided their own definitions of quality care. The resulting themes and subthemes are discussed in detail below.

\section{Clinic infrastructure}


Providers from facilities with a newer clinic infrastructure identified this as an indicator of quality care. Providers from other facilities mentioned infrastructure at their clinics as an indicator of poor quality.

\section{Lack of resources}

Lack of resources in the clinic, including medication, equipment, cleaning supplies, and staff were all associated with less ability to provide quality care. Shortage of medication was cited as an indicator of care quality, with providers from three different facilities discussing how their lack of medication was indicative of low-quality care. Providers also discussed how the lack of medical equipment meant that they could not provide quality care to their patients:

"I think what can help me to provide good quality care or to do my work well is when I have equipment. We don't have enough but with the little that we have, we are trying." (Clinic 5, professional nurse)

Some providers also spoke about staff shortages and how it impacted their ability to provide quality care. Other providers spoke of resource availability more generally as an indication of good quality care and a source of pride.

\section{Respectful care}

Providers cited positive staff behavior as an indicator of quality, including communicating in a positive and open manner, explaining treatments, and conducting adequate counselling. Conversely, providers who reported colleagues having "attitudes" or showing lack of empathy for patients indicated that their care was of poor quality. Respectful care also included maintaining patient confidentiality. Some providers discussed confidentiality as a factor enabling patients to come to clinic and adhere to medication.

"When it comes to HIV patients and confidentiality, we are providing high quality compared to other clinics of Bushbuckridge. We are the best and I know that." (Clinic 9, professional nurse)

\section{Time spent in the clinic}

Time spent in the clinic, including short waiting times for services and longer face-to-face visits with providers, were seen as indicators of quality care. Some clinics with short wait times credited the central chronic medication dispensing and distribution (CCMDD) program (part of the national differentiated care facility decongestion initiative) for their ability to provide quicker service to HIV-positive patients:

"I would say our clinic is the best when comes to treating patients who are HIV positive. Particularly the chronic [care]...the treatment is packed with the [recipient's] name on the outside of the package... They don't stay for more than an hour." (Clinic 1 (CHC), lay counsellor)

Providers also cited their lack of ability to spend time with patients as an indicator of poor quality. Some discussed how they did not have enough staff to attend to the high patient volume, leading to long wait times and rushed care. Several providers discussed how time constraints led to diminished or complete 
lack of counseling, including on how to take their antibiotics properly, or HIV counseling and testing; or skipping procedures like Pap smears or getting sputum samples.

\section{Adherence to clinical guidelines}

Providers discussed the importance of knowing and adhering to clinical guidelines, and of attending district-supported trainings and workshops in informing quality patient care.

\section{Service volume}

Some providers reported using patient and service (i.e. number of HIV tests or Pap smears) data to determine quality. One provider interpreted clinic data showing low utilization rates as a sign of poor quality:

"Our utilization statistics are also low; this is proof that we don't provide good quality care. If we were, we would have higher numbers." (Clinic 5, professional nurse)

\section{Patient data are not utilized as indicators of quality}

Patient data were not broadly discussed as indicators of quality care. A few providers identified patient utilization of the facility, particularly by patients from other villages who may be bypassing their nearest healthcare facility, as an indicator of quality care. Providers also discussed seeing patients' health improve after receiving treatment from their facility as an indicator of quality care.

\section{Mitigating strategies}

While challenges in providing quality care have been documented, less on how providers overcome these barriers has been documented. An emergent theme in our analysis of the data were mitigating strategies that providers used to combat challenges to address barriers to providing quality care.

\section{Reallocation of resources within the clinic}

To address understaffing, providers took shorter lunch breaks and split staff into teams to balance leave days. To maximize clinic space and maintain confidentiality, several facilities moved their lay counsellors to a space that could be devoted to HIV counselling and testing, such as a mobile unit, the meeting room, or the nurses' accommodation on site. Facilities with more space also recommended designating one building for CCMDD both to keep the queue moving quickly and to streamline treatment pickup for all chronic patients.

\section{Sharing resources across clinics}

To make up for resource shortages, providers shared equipment across facilities, bought their own supplies (i.e. batteries, soap, toilet paper), and sometimes went as far as driving a patient to the hospital in their own car if an ambulance was not available. Many providers expressed a sense of duty to help 
their patients, despite the shortage of resources and personal costs. Providers also tried to circumvent the system by ordering quantities of medication greater than the expected patient population, sharing medication between facilities, or prescribing medications in smaller quantities at a time than recommended by guidelines (i.e. one month of ART instead of three, in order to supply more patients). Providers in the Agincourt HDSS area discussed using a WhatsApp group across facilities to discuss supply of ART in particular:

With HIV treatment [stock-outs] were not happening as we are trying by all means to ask for it from nearby clinics. They are assisting us. We have a WhatsApp group that we use to talk to each other. If we have a shortage of this treatment, we will WhatsApp it so everyone in our group will know. (Clinic 5 , professional nurse)

\section{Discussion}

This qualitative study of providers in rural PHC facilities in South Africa elucidated their definitions of high-quality care, the barriers they face in providing care, and the mitigating strategies they employ in response to these barriers. Definitions of quality were focused on structure and resources, as well as some process elements - patient experience and competent care. Few providers identified patient outcomes like treatment success or retention in treatment as indicators of quality. In identifying barriers to high quality care, providers linked the deficiencies in infrastructure and support to deficits in care delivery, such as long wait times and short visits due to limited staffing or privacy breaches due to insufficient space to maintain confidentiality. Finally, providers identified mitigating strategies such as coordination across clinics to address medication shortages in individual facilities. Interwoven throughout the providers' discussion was the poor communication between the district, PHC supervisors, and implementers at the facility level. This manifested in myriad ways; for example, lack of responsiveness from the depot in regards to medication stock-outs at the clinics, or a training model that did not meet providers needs.

The disconnect between national policy and clinic-level implementation was further highlighted in discussions with providers around indicators of quality. Although the ICRM program was implemented across South Africa in 2016, collection and utilization of these indicators does not appear to be ongoing in rural PHC clinics in the Agincourt HDSS. Throughout the interview process, there were challenges clearly defining the concept of metrics and indicators in both English and Shangaan. The concept of quality measurement did not seem to resonate with providers, even though they could discuss at length the quality challenges they observed. Patient outcome metrics were largely not addressed, although some providers discussed service numbers (such as HTS), despite the national push to gather data. There is a demonstrated gap in translation from the metrics that are being collected and pushed to the provincial and national departments of health to what providers are using on the ground. This is reflected by a 2019 study conducted in Gauteng and Mpumalanga which found that PHC facility managers from these provinces reported lack of involvement in conceptualization of the ICRM program.(34) For quality improvement efforts to be meaningful, there is a need for data literacy education and training at the clinic 
operational and provider level, involvement in development of indicators, and a clear feedback loop allowing what providers see as critical information to be reflected in the national quality measurement strategy.

Providers discussed many barriers to providing quality care, which fell under the foundational categories of workforce and tools. These barriers were all tied back to lack of human and material resources available to the facilities, which impacted every element of care. Understaffing, need for additional training, poor facility infrastructure, lack of clinical care space and resources, and the challenges in providing care associated with these deficiencies have previously been documented in South Africa. Our research frames these gaps in terms of foundational elements of quality care, and the strategies providers use to complete their duties in spite of them, in order to highlight the need for attention and intervention at the meso (district) and macro (province/national) levels to equip PHC facilities with the resources they need.

The first major barrier identified was understaffing. Previous research in South Africa has identified shortage of human resources $(2,8,18)$ and high patient-staff ratios $(35)$ as causes of service delivery issues, including a study conducted with health systems stakeholders in the Agincourt HDSS.(17) It has been noted that health workers' heavy workload is a reason for both short- and long-term sickness absence (22), and that among health care providers in sub-Saharan Africa, burnout is highest among nurses and is associated with their work environments.(36) In previous research, $42 \%$ of providers in the Agincourt HDSS surveyed reported planning to leave their job within the next two years (in some clinics, up to $81 \%$ of staff).(37) Our research supports these findings that facilities are understaffed, leading to over-crowding, rushed or incomplete services, and negatively impacting providers' health and wellbeing. Providers across the nine facilities discussed making referrals to hospitals or other PHC facilities as a common mitigating strategy for addressing shortages of staff, medical supplies, and medications. Referring patients to other facilities experiencing similar staffing shortages and lacking resources can only exacerbate these existing service delivery issues. This practice can also consume patient time and money, and affect retention in care, as it may be an effort to get to the second facility, or patients may choose not to follow the referral. Increasing the number of lower-level nurses as well as cleaners, file clerks and data capturers could facilitate more effective task-shifting and allow higher-level nurses to focus on their clinical duties.

Providers also discussed challenges with scheduling throughout the week, including leave and training days, as contributing to their heavy workload. Another study from the MRC/Wits-Agincourt Unit identified the practice of holding clinics in the mornings leading to flooding the facilities at certain times of day and exacerbating staffing problems. Those researchers suggested making flexible appointments as a no-cost measure to reduce such crowding (17); however, this would require providing additional staff to monitor appointments as well as the materials and space required to support this approach. Another strategy for improvement would be to allocate staff more evenly throughout the week, including weekends, rather than focusing staffing on Tuesdays-Thursdays as discussed in our research. 
The second major barrier was staff feeling they did not receive enough skills training. Our research illuminates how the current system for training is not responsive to providers' needs and realities. Other work in the Agincourt HDSS has shown a need for additional training on HIV treatment as prevention (38), and providers in this study discussed a need for trainings in skills and new treatment guidelines. The current training model, where one senior staff member is sent to a training and then expected to disseminate information to the rest of the staff, does not appear to meet providers' needs. Sending a master trainer to provide in-service trainings in the clinics themselves could be a viable option to address the challenge of knowledge dissemination without taking providers away from their facilities. If online trainings are a route to be explored, then data for providers to access internet on their cell phones should be distributed to providers as well.

Poor facility infrastructure was the third major barrier identified to providing quality care. Healthcare associated infections have been noted in past research due to aging infrastructure and inadequate environmental cleaning.(35) Other research has found poor hygiene and infection control in South African healthcare facilities. $(2,19)$ Structural issues noted by providers such as leaking roofs, collapsed ceilings, insect and bat infestations, all require immediate attention and maintenance to ensure safe environments for providers and patients. One provider felt the poor infrastructure was indicative of the South African government's lack of attention to rural areas. Indeed, problems with facility infrastructure in the Agincourt HDSS may date back to apartheid, when the area was a "homeland" with a smaller population, in which limited and poor quality services were provided.(17) These facilities must now combat deteriorating infrastructure and growing populations. Mpumalanga Department of Health stakeholders have noted that facility maintenance is not within their control, but rather falls under the provincial Department of Public Works, Roads, and Transport (17), thus requiring hard to attain crossdepartmental coordination.

The fourth major barrier identified was a chronic lack of equipment, medication, and other resources in the facilities, also documented in previous studies in South Africa $(2,35,39)$ and within the Agincourt HDSS study area. $(40,41)$ A 2018 study intervention to improve hypertension care in PHCs in the Agincourt HDSS was unsuccessful, in part due to unreliable blood pressure machines and cuffs, intermittent drug shortages, and lack of space.(40) Mitigating strategies to combat this lack of resources, such as relying on peer-provider networks and drug substitution (39) are not sufficient to address the problem of chronic medication shortages. Even when facilities are able to share, providers may often have to use their own money for transport to pick them up. Some providers fear that giving shorter doses of medication such as ART is problematic for patients who may have to travel far and at personal expense to get to the facility, and that it may deter them from coming back. There is an evident need for better coordination between facilities and the depot, and between the depot and their suppliers.

The limitations of this study should be addressed. This research was conducted in a rural sub-district of Mpumalanga, and our findings may not be generalizable to other districts or provinces throughout South Africa. An additional limitation of this research is that we did not interview a random sample of providers, 
and provider availability was subject to limitations based on clinical responsibility. However, we were able to interview approximately 1 in 6 providers across the 9 clinics in the Agincourt research area.

\section{Conclusion}

PHC providers in the Agincourt HDSS study area are faced with significant barriers to providing quality care driven by budget constraints and underfunding, which lead to deficits in workforce, staffing, tools and resources. These barriers to providing care are compounded by the disconnect between policymakers and implementation, and systems that are unresponsive to their needs. These findings demonstrate that provider perspectives must be integrated within quality improvement planning programs to ensure these efforts start at provincial and district levels to provide a sound basis for facility-level improvement. Improvements targeted at individual facilities and providers alone would not appress providers' concerns and daily challenges.

Given the breadth of existing evidence documenting shortages in foundations of quality care at PHC facilities in South Africa, future research should include providers as partners in quality improvement efforts, letting their perspectives inform initiatives and planning. The issues in resource allocation and support and supervision identified fit with the Lancet Global Health Commission's recommendations, and other research conducted in Mpumalanga Province (42), for health systems to focus less on individual provider/clinic interventions, and try more meso (district) and macro (provincial/national) approaches. (12) This research elucidates the gaps in foundations of quality care; serving as a reminder that new clinic-level programs or initiatives are unlikely to succeed until the cracks in these foundations are addressed by the district and provincial Departments of Health.

\section{List Of Abbreviations}

AIDS - acquired immunodeficiency syndrome

ART - antiretroviral therapy

CCMDD - central chronic medication dispensing and distribution

$\mathrm{CHC}$ - community health centers

HDSS - Health and Socio-Demographic Surveillance System

HIV - human immunodeficiency virus

HTS - HIV testing services

MRC - Medical Research Council (South Africa)

$\mathrm{PHC}$ - primary health care 


\section{Declarations}

\section{Ethics approval and consent to participate}

All procedures performed in studies involving human participants were in accordance with the ethical standards of the relevant institutional and national research committees and with the 1964 Helsinki declaration and its later amendments or comparable ethical standards. Approval for this research was provided by the Harvard Human Research Protection Program (IRB18-1400), the Human Research Ethics Committee (Medical) at the University of the Witwatersrand, Johannesburg (Ethics Ref No. 150104), and the Provincial Health Research Committee at the Mpumalanga Province Department of Health (MP_201812_004).

Informed consent was obtained from all individual participants included in the study.

\section{Consent for publication}

Not applicable.

\section{Availability of data and materials}

The datasets used and/or analyzed during the current study are available from the corresponding author on reasonable request.

\section{Competing interests}

Dr. Leslie discloses research support from the Bill \& Melinda Gates Foundation, the World Health Organization, and the World Bank during the conduct of this research. The authors declare that they have no competing interests.

\section{Funding}

Research undertaken at the MRC/Wits Rural Public Health and Health Transitions Research Unit site in Mpumalanga was supported by the United States National Institute of Mental Health (R01MH087118 Pettifor, R01MH103198 - Lippman/Pettifor). The MRC/Wits-Agincourt Unit and HDSS is supported by the South African Medical Research Council, the University of the Witwatersrand and the Wellcome Trust, United Kingdom. The qualitative interviews and related analyses conducted for this manuscript were supported by Harvard University Center for AIDS Research (CFAR), an NIH-funded program (P30AI060354).

\section{Authors' contributions}

Conceptualization: SA, KK, RT, HHL. Data collection: RW, MM. Formal Analysis: RW. Writing - original draft: RW, HHL. Writing - review \& editing: RW, SA, RT, KK, MM, HHL 
We are grateful to the healthcare providers in the Agincourt HDSS for participating in this research.

\section{References}

1. Kahn K. Population health in South Africa: Dynamics over the past two decades. J Public Health Policy. 2011;32(S1):S30-36.

2. Maphumulo WT, Bhengu BR, Maphumulo W. Challenges of quality improvement in the healthcare of South Africa post-apartheid: A critical review. Open Access. 2019;9.

3. Mayosi BM, Lawn J, van Niekerk A, Bradshaw D, Abdool Karim SS, Coovadia HM. Health in South Africa: changes and challenges since 2009. The Lancet. 2012;380(9858):2029-43.

4. Pillay-van Wyk V, Msemburi W, Laubscher R, Dorrington RE, Groenewald P, Glass T, et al. Mortality trends and differentials in South Africa from 1997 to 2012: second National Burden of Disease Study. Lancet Glob Health. 2016 Sep;4(9):e642-53.

5. Ameh S, Gómez-Olivé FX, Kahn K, Tollman SM, Klipstein-Grobusch K. Predictors of health care use by adults 50 years and over in a rural South African setting. Glob Health Action. 2014 Dec;7(1):24771.

6. Uwimana J, Jackson D, Hausler H, Zarowsky C. Health system barriers to implementation of collaborative TB and HIV activities including prevention of mother to child transmission in South Africa: Health system barriers to TB and HIV care. Trop Med Int Health. 2012 May;17(5):658-65.

7. Winchester MS, King B. Decentralization, healthcare access, and inequality in Mpumalanga, South Africa. Health Place. 2018 May;51:200-7.

8. Coovadia H, Jewkes R, Barron P, Sanders D, Mclntyre D. The health and health system of South Africa: historical roots of current public health challenges. The Lancet. 2009 Sep;374(9692):817-34.

9. van Rensburg HC. South Africa's protracted struggle for equal distribution and equitable access still not there. Hum Resour Health. 2014 Dec;12(1):26.

10. Meyer JC, Schellack N, Stokes J, Lancaster R, Zeeman H, Defty D, et al. Ongoing Initiatives to Improve the Quality and Efficiency of Medicine Use within the Public Healthcare System in South Africa; A Preliminary Study. Front Pharmacol [Internet]. 2017 Nov 9 [cited 2019 May 23];8. Available from: https://www.ncbi.nlm.nih.gov/pmc/articles/PMC5677783/

11. Matsoso MP, Hunter JR, Brijlal V. Embedding quality at the core of universal health coverage in South Africa. Lancet Glob Health. 2018 Nov;6(11):e1153-4.

12. Kruk ME, Gage AD, Arsenault C, Jordan K, Leslie HH, Roder-DeWan S, et al. High-quality health systems in the Sustainable Development Goals era: time for a revolution. Lancet Glob Health. 2018 Nov;6(11):e1196-252.

13. Mogashoa, Monene Grysel, Pelser, Gert Petrus Jacobus. An analysis of the implementation of the national core standards in public hospitals. Afr Insight. 2014;44(2):142-7.

14. Moyakhe NP. Quality healthcare: An attainable goal for all South Africans? South Afr J Bioeth Law. 2014 Nov 4;7(2):80. 
15. Ideal Clinic Manual Version 18 [Internet]. Pretoria: National Department of Health; 2018. Available from: https://www.idealhealthfacility.org.za/docs/v18/Final\%20ldeal\%20Clinic\%20Manual\%20\%20version\%2018\%20(26\%20July\%202018).pdf

16. Kruk ME, Gage AD, Joseph N, Danaei G, Garcia-Saiso S, Salomon J. Mortality due to low quality health systems in the Universal Health Coverage era: a systematic analysis of amenable deaths in 137 countries. The Lancet. 2018;392(10160):2203-12.

17. D’Ambruoso L, van der Merwe M, Wariri O, Byass P, Goosen G, Kahn K, et al. Rethinking collaboration: developing a learning platform to address under-five mortality in Mpumalanga province, South Africa. Health Policy Plan. 2019 Jul 1;34(6):418-29.

18. Tana V. "Experiences of chronic patients about long waiting time at a community health centre in the Western Cape" [Internet] [Master of Nursing Sciences, Faculty of Health]. [South Africa]: University of Stellenbosch; 2013. Available from: https://pdfs.semanticscholar.org/803e/58cac7e0cc2c646fbb167cc9270978bbc941.pdf

19. Dunjwa M. Public health facilities audit results: Office of Health Standards Compliance (OHSC) briefing [Internet]. Pretoria, South Africa: Parliamentary Monitoring Group; 2016. Available from: https://pmg.org.za/committee-meeting/22233/

20. Nuwagaba-Biribonwoha H, Mayon-White RT, Okong P, Carpenter LM. Challenges faced by health workers in implementing the prevention of mother-to-child HIV transmission (PMTCT) programme in Uganda. J Public Health. 2007 Jul 17;29(3):269-74.

21. Schuster RC, McMahon DE, Young SL. A comprehensive review of the barriers and promoters health workers experience in delivering prevention of vertical transmission of HIV services in sub-Saharan Africa. AIDS Care. 2016 Jun 2;28(6):778-94.

22. Belita A, Mbindyo P, English M. Absenteeism amongst health workers - developing a typology to support empiric work in low-income countries and characterizing reported associations. Hum Resour Health. 2013 Dec;11(1):34.

23. Medley AM, Kennedy CE. Provider Challenges in Implementing Antenatal Provider-Initiated HIV Testing and Counseling Programs in Uganda. AIDS Educ Prev. 2010;22(2):87-99.

24. Doherty TM, McCoy D, Donohue S. Health system constraints to optimal coverage of the preven- tion of mother-to-child HIV transmission programme in South Africa: lessons from the implementation of the national pilot programme. 2005;5(3):6.

25. Rujumba J, Tumwine JK, Tylleskär T, Neema S, Heggenhougen HK. Listening to health workers: lessons from Eastern Uganda for strengthening the programme for the prevention of mother-to-child transmission of HIV. BMC Health Serv Res. 2012 Dec;12(1):3.

26. Sprague C, Chersich MF, Black V. Health system weaknesses constrain access to PMTCT and maternal HIV services in South Africa: a qualitative enquiry. AIDS Res Ther. 2011;8(1):10.

27. Kahn K, Collinson MA, Gomez-Olive FX, Mokoena O, Twine R, Mee P, et al. Profile: Agincourt Health and Socio-demographic Surveillance System. Int J Epidemiol. 2012 Aug 1;41(4):988-1001. 
28. Gómez-Olivé FX, Angotti N, Houle B, Klipstein-Grobusch K, Kabudula C, Menken J, et al. Prevalence of HIV among those 15 and older in rural South Africa. AIDS Care Psychol Socio-Med Asp AIDSHIV. 2013;25(9):1122-8.

29. Manne-Goehler J, Montana L, Gómez-Olivé FX, Rohr J, Harling G, Wagner RG, et al. The ART Advantage: Health Care Utilization for Diabetes and Hypertension in Rural South Africa. J Acquir Immune Defic Syndr 1999. 2017 15;75(5):561-7.

30. Lippman SA, Pettifor A, Rebombo D, Julien A, Wagner RG, Kang Dufour M-S, et al. Evaluation of the Tsima community mobilization intervention to improve engagement in HIV testing and care in South Africa: study protocol for a cluster randomized trial. Implement Sci. 2017 Dec;12(1):9.

31. Donabedian A. The Quality of Care: How Can It Be Assessed? JAMA. 260(12):1743-8.

32. Green J, Thorogood N. Qualitative Methods for Health Research. 4th ed. London: SAGE Publications Ltd; 2018. 289-96 p.

33. Rohleder P, Swartz L. "What l've noticed what they need is the stats": Lay HIV counsellors' reports of working in a task-orientated health care system. AIDS Care. 2005;17(3):397-406.

34. Muthathi IS, Levin J, Rispel LC. Decision space and participation of primary healthcare facility managers in the Ideal Clinic Realisation and Maintenance programme in two South African provinces. Health Policy Plan. 2020 Apr 1;35(3):302-12.

35. Dramowski A, Cotton MF, Whitelaw A. A framework for preventing healthcare-associated infection in neonates and children in South Africa. S Afr Med J. 2017 Feb 27;107(3):192.

36. Dubale BW, Friedman LE, Chemali Z, Denninger JW, Mehta DH, Alem A, et al. Systematic review of burnout among healthcare providers in sub-Saharan Africa. BMC Public Health. 2019 Dec;19(1):1247.

37. West R, Leslie HH, Gomez-Olive FX, Kahn K, Twine R, Filiatreau L, et al. Using multiple modes of assessment to measure patient experience in public clinics in rural Mpumalanga, South Africa. South Africa AIDS Conference; 2019 Jun 12; Durban, South Africa.

38. Lippman SA, West R, Gómez-Olivé FX, Leslie HH, Twine R, Gottert A, et al. Treatment as PreventionProvider Knowledge and Counseling Lag Behind Global Campaigns: JAIDS J Acquir Immune Defic Syndr. 2020 Feb;83(2):e9-12.

39. Zakumumpa H, Kiweewa FM, Khuluza F, Kitutu FE. "The number of clients is increasing but the supplies are reducing": provider strategies for responding to chronic antiretroviral (ARV) medicines stock-outs in resource-limited settings: a qualitative study from Uganda. BMC Health Serv Res. 2019 Dec;19(1):312.

40. Goudge J, Chirwa T, Eldridge S, Gómez-Olivé FXF, Kabudula C, Limbani F, et al. Can lay health workers support the management of hypertension? Findings of a cluster randomised trial in South Africa. BMJ Glob Health. 2018 Feb;3(1):e000577.

41. Limbani F, Thorogood M, Gómez-Olivé FX, Kabudula C, Goudge J. Task shifting to improve the provision of integrated chronic care: realist evaluation of a lay health worker intervention in rural South Africa. BMJ Glob Health. 2019 Jan;4(1):e001084. 
42. Schneider H, George A, Mukinda F, Tabana H. District Governance and Improved Maternal, Neonatal and Child Health in South Africa: Pathways of Change. Health Syst Reform. 2020 Jan 1;6(1):e1669943. 